\title{
Técnicas de aprendizaje automático en el diagnóstico de aerogeneradores
}

\section{Machine learning techniques in the diagnosis of wind turbines}

\author{
GARCÍA, Uriel A. †*, IBARGÜENGOYTIA, Pablo H., DÍAZ-GONZÁLEZ, Lorena y HERMOSILLO- \\ VALADEZ, Jorge
}

Instituto Nacional de Electricidad y Energías Limpias. Reforma 113, Palmira. 62490 Cuernavaca, Morelos

ID 1 ${ }^{\mathrm{er}}$ Autor: Uriel A, García / CVU CONACYT ID: 862399

ID 1 ${ }^{\text {er }}$ Coautor: Pablo H, Ibargüengoytia / ORC ID: 0000-0001-8893-6708, CVU CONACYT ID: 120007

ID $2^{\text {do }}$ Coautor: Lorena, Díaz González / ORC ID: 0000-0003-1577-5629, CVU CONACYT ID: 174039

ID $3^{\text {er }}$ Coautor: Jorge, Hermosillo Valadez / ORC ID: 0000-0001-9040-767X

DOI: $10.35429 / J R E .2019 .11 .3 .7 .14$

Recibido 13 de Octubre, 2019; Aceptado 08 Diciembre, 2019

\begin{abstract}
Resumen
EL Centro Mexicano de Innovación en Energía Eólica (CEMIE-Eólico) diseñó un sistema de diagnóstico de aerogeneradores basado en modelos de comportamiento de la turbina utilizando las señales del sistema de control supervisorio y adquisición de datos (SCADA por sus siglas en inglés). El sistema proporciona un patrón de variables que presentan comportamiento anormal en presencia de una falla. Los patrones se forman con la detección del comportamiento anormal de las variables durante una ventana de tiempo en que se manifiesta la falla. En este trabajo, se presenta la aplicación de técnicas de aprendizaje automático para la identificación de fallas en aerogeneradores después del sistema de diagnóstico. Los datos de entrenamiento y validación se obtuvieron a partir de la simulación de seis diferentes fallas en el aerogenerador usando la Máquina Eólica Mexicana (MEM) diseñada en el Instituto Nacional de Electricidad y Energías Limpias (INEEL). Se aplicó el sistema de diagnóstico, se generaron los perfiles de comportamiento anormal y se realizaron experimentos para la clasificación multiclase de patrones de fallas usando el algoritmo de "Random Forest". Finalmente, se evaluó el desempeño del algoritmo usando las métricas de exactitud y precisión logrando un $91 \%$ en la clasificación de patrones para identificar la falla raíz.
\end{abstract}

Diagnóstico de aerogeneradores, Random Forest, Aprendizaje automático

\begin{abstract}
The Mexican Center for Innovation in Wind Energy (CEMIE-Eólico) designed a wind turbine diagnostic system based on turbine behavior models using the signals of the Supervisory Control and Data Acquisition system (SCADA). The system provides a pattern of variables that exhibit abnormal behavior in the presence of a fault. The patterns are formed with the detection of the abnormal behavior of the variables during a time window in which the failure manifests itself. This paper presents the application of machine learning techniques for the identification of faults in wind turbines after the diagnostic system. The training and validation data were obtained from the simulation of six different faults in the wind turbine using the Mexican Wind Machine (MEM) designed at the National Institute of Electricity and Clean Energy (INEEL). The diagnostic system was applied, profiles of abnormal behavior were generated and experiments were carried out for the multiclass classification of fault patterns using the "Random Forest" algorithm. Finally, the algorithm performance was evaluated using accuracy and precision metrics achieving $91 \%$ in the classification of patterns to identify the root failure.
\end{abstract}

Wind turbine diagnosis, Random Forest, Machine learning

Citación: GARCÍA, Uriel A., IBARGÜENGOYTIA, Pablo H., DÍAZ-GONZÁLEZ, Lorena y HERMOSILLO-VALADEZ, Jorge. Técnicas de aprendizaje automático en el diagnóstico de aerogeneradores. Revista de Energías Renovables. 2019 311: 7-14

\footnotetext{
*Correspondencia al Autor (Correo Electrónico: uriel.garcia@gmail.com)
}

$\uparrow$ Investigador contribuyendo como primer autor. 


\section{Introducción}

Existe una necesidad urgente de realizar una transición hacia el uso de recursos renovables para la generación de potencia eléctrica a nivel mundial. Todos los países, en mayor o menor grado hacen esfuerzos para lograr esa transición. México no es la excepción.

Según la Asociación Mexicana de Energía Eólica (AMDEE), en el 2018 existían 2,447 aerogeneradores (AG) formando parte de 54 parques eólicos y con una generación total de 4,935 Mega Watts, resultando en un 5.08\% de la producción nacional (AMDEE, 2017).

Dada la creciente importancia de la energía eólica en la generación total nacional y dado que un $\mathrm{AG}$ tiene un tiempo de vida promedio entre 20 y 25 años, la necesidad de contar con sistemas de diagnóstico de AGs resulta evidente. Lograr la identificación de fallas incipientes podrá lograr acciones de mantenimiento y reparación que permitan elevar los índices de confiabilidad y disponibilidad de los parques eólicos.

Para atender las necesidades del sector eólico en México, la secretaría de Energía (SENER) y el CONACYT, crearon el CEMIEEólico a través del Fondo Sectorial SENERSustentabilidad. El proyecto reportado en este artículo es resultado de las actividades en el proyecto P12 del CEMIE-Eólico. La idea fue utilizar técnicas de Inteligencia Artificial (IA) en apoyo al sector eólico. En este proyecto se diseñó un sistema de diagnóstico utilizando modelos probabilistas de comportamiento para poder detectar desviaciones a ese comportamiento normal.

La literatura reporta diferentes formas de atacar el problema de diagnóstico de aerogeneradores (AG). La mayoría de los trabajos consultados pertenecen a la comunidad de Monitoreo de la Condición (Condition monitoring, CM, en inglés). El artículo de revisión de literatura (García Márquez, MarkTobias, Pinar Pérez, \& Papaelias, 2012) establece los métodos más utilizados en el monitoreo de la condición o diagnóstico. Ellos son: i) análisis de vibraciones, ii) análisis de emisiones acústicas, iii) técnicas de pruebas ultrasónicas, iv) análisis en aceite, v) medición del esfuerzo o tensión en aspas, vi) termografía, vii) métodos de pulso de choque, viii) inspecciones radiográficas y otros métodos.
Sin embargo, esos métodos tradicionales han requerido siempre personal experto en el área y la formación de modelos muy complejos y difíciles de construir y mantener. Alternativamente, en la actualidad existen métodos computacionales basados en técnicas de inteligencia artificial para realizar el diagnóstico. Como un ejemplo, el trabajo reportado en (Zhou, Yu, \& Zhang, 2015) está basado en ontologías y en análisis crítico de efectos de modos de falla (FEMCA por sus siglas en inglés). Con este análisis, la metodología reportada genera una ontología y crea una base de conocimientos para alimentar un sistema experto. El problema de este método y en general con los sistemas expertos es que no manejan la incertidumbre que es común en esta aplicación. Otro trabajo reportado en (Meik, Ferreira Santos, \& Achiche, 2013) utiliza modelos de comportamiento de la turbina y datos históricos del SCADA. El enfoque utilizado es de sistemas de inferencia neurodifusa adaptada (ANFIS por sus siglas en inglés) pero no se tiene ninguna distinción de los modos de operación de la turbina eólica.

El trabajo reportado en este artículo está formado por dos etapas. La primera utiliza modelos del comportamiento para poder detectar desviaciones al comportamiento normal. Los modelos de comportamiento se basan en redes Bayesianas (Pearl, 1988) que capturan las relaciones probabilistas entre las variables del SCADA. La salida de la primera etapa consiste en un conjunto de variables que presentan un comportamiento anormal según los modelos de comportamiento. La segunda etapa toma el conjunto de variables con comportamiento anormal y forma un patrón de falla. El objetivo del trabajo es reconocer los patrones de falla generados en el sistema de diagnóstico y lograr la identificación de la falla raíz utilizando técnicas de aprendizaje de máquina. La metodología seguida consistió en la utilización de un simulador de aerogenerador para realizar corridas experimentales del aerogenerador funcionando correctamente y después se insertaron 6 fallas diferentes. De acuerdo a los patrones generados en los experimentos, se usó el algoritmo de "Random Forest" para crear el modelo de identificación de fallas raíces. La principal contribución del trabajo reportado en el artículo es la aplicación del Random Forest para aprender el patrón de las fallas y la medición del desempeño que tuvo este algoritmo en esta identificación de fallas raíces.

GARCÍA, Uriel A., IBARGÜENGOYTIA, Pablo H., DÍAZ GONZÁLEZ, Lorena y HERMOSILLO-VALADEZ, Jorge. Técnicas de aprendizaje automático en el diagnóstico de aerogeneradores. Revista de Energías Renovables. 2019 
Este artículo se organiza de esta manera: La siguiente sección describe el sistema de diagnóstico de aerogeneradores construido en el proyecto del CEMIE-Eólico. Se denomina DxAG. La sección 3 describe la Máquina Eólica Mexicana (MEM) con la cual se realizaron los experimentos. La sección 4 describe los experimentos y resultados realizados con un simulador de la MEM, y finalmente, la sección 5 concluye este artículo y propone tareas para mejorar el sistema DxAG.

\section{Diagnóstico de AeroGeneradores, DxAG}

El sistema de diagnóstico de aerogeneradores DxAG fue desarrollado en el CEMIE-Eólico. La idea es crear modelos del comportamiento del AG para poder identificar desviaciones al comportamiento normal aprendido (Ibargüengoytia, García, Reyes, \& Borunda, 2016). Los modelos de comportamiento se representan con una red Bayesiana donde se codifican las relaciones probabilistas entre las señales del SCADA. La detección de desviaciones al comportamiento normal se hace utilizando la teoría de validación de información (Ibargüengoytia, Vadera, \& Sucar, 2006).

La Figura 1 muestra el papel que realiza el sistema DxAG en el presente trabajo (García, Ibargüengoytia, Reyes, \& Borunda, 2016).

Una vez aprendidos los modelos de comportamiento con datos históricos del SCADA, se tiene al DxAG en condiciones de operación. Para su funcionamiento, se conecta al SCADA con el DxAG para adquirir la información actualizada de las variables, cada cierto tiempo de muestreo, por ejemplo cada 5 minutos. El DxAG estima el valor de cada variable infiriéndola en el modelo probabilista de comportamiento y lo compara con la lectura del valor real de la variable en turno. Si el valor real coincide con el valor inferido en una distribución de probabilidad, entonces se considera comportamiento normal.

En caso de que no correspondan los valores, se supone una variable con comportamiento anormal en las condiciones de operación correspondientes. Cuando se realiza este procedimiento de inferencia-comparación de todas las variables, se genera un patrón de comportamiento de falla como se muestra a la derecha de la Figura 1.
El patrón de falla resultante se puede ver como un video de una ventana de tiempo donde se muestran las variables (cada una con diferente color) que presentaron comportamiento anormal. Sin embargo, esos patrones sólo indican que hubo una situación anormal, pero no se puede identificar la falla raíz. La sección 4 explica en detalle el uso de la técnica de aprendizaje automático para el reconocimiento de patrones de fallas raíces.

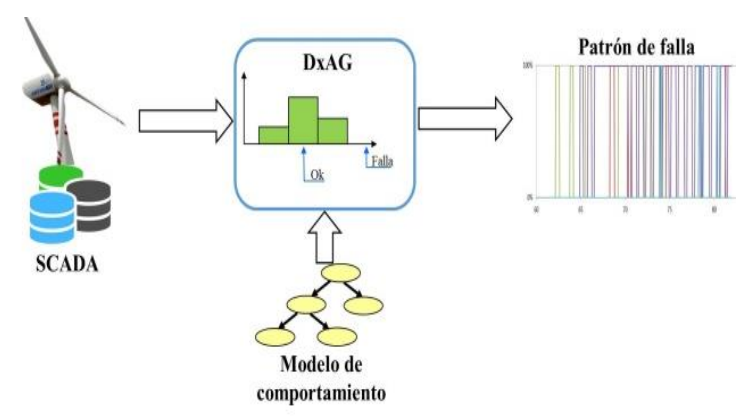

Figura 1 Arquitectura para generar patrones de fallas Fuente: Elaboración Propia

\section{Máquina Eólica Mexicana (MEM)}

La Máquina Eólica Mexicana es desarrollada actualmente por el (INEEL) con el propósito de aprovechar las oportunidades que ofrece el mercado eólico internacional. La MEM es un aerogenerador de eje horizontal con control de potencia por cambio de ángulo de paso, de velocidad variable, conformado por un rotor de 3 aspas diseñado con las siguientes características de operación:

\section{- $\quad$ Potencia nominal de 1.2 MW \\ - $\quad$ Diámetro del rotor de $60 \mathrm{~m}$ \\ - $\quad$ Velocidad de inicio de $4 \mathrm{~m} / \mathrm{s}$ \\ - Velocidad de paro de $25 \mathrm{~m} / \mathrm{s}$}

Actualmente, el aerogenerador cuenta con un diseño de funcionamiento simulado en la herramienta de computacional Focus6 (WMC, 2010).

Focus6 es una herramienta modular integrada para diseñar aerogeneradores $y$ componentes de la misma, tales como palas de rotor y soporte.

Esta herramienta de simulación proporciona una interfaz de usuario consistente que integra una serie de herramientas para simular el funcionamiento normal o con falla del aerogenerador. 
Para construir un modelo de comportamiento normal de la MEM, el simulador se configuró con las siguientes características: se generaron simulaciones de 5 min con una frecuencia de 16 muestras por segundo de 96 variables, se realizaron simulaciones con un rango de velocidades de 12$15 \mathrm{~m} / \mathrm{s}$ y 300 variaciones de velocidades dentro del rango definido. Para la simulación de 6 fallas (ver Tabla 1) insertadas al aerogenerador se usaron las mismas características de operación que se utilizaron para generar el modelo de comportamiento normal, se agregó una ventana de tiempo de 10 segundos por cada una de las fallas simuladas y se estableció que la falla debe de iniciar en el segundo 30.

\begin{tabular}{|l|l|}
\hline \multicolumn{1}{|c|}{$\begin{array}{c}\text { Id } \\
\text { Falla }\end{array}$} \\
\hline F0 & Operación normal \\
\hline F1 & $\begin{array}{l}\text { Sin velocidad de Pitch en todas las aspas } \\
\text { (control suprimido) }\end{array}$ \\
\hline F2 & $\begin{array}{l}\text { Exceso de velocidad de Pitch en todas las } \\
\text { aspas (falla de sensor) }\end{array}$ \\
\hline F3 & Sin velocidad de Pitch en aspa No. 2 \\
\hline F4 & Pitch fuera de control en aspa No. 2 \\
\hline F5 & Cortocircuito en el generador \\
\hline F6 & Falla en el sistema Yaw \\
\hline
\end{tabular}

Tabla 1 Fallas insertadas en la MEM

Las fallas F1, F2, F3 y F4 son fallas referentes al Pitch. El pitch es el control que hace girar las aspas para que puedan presentar la máxima resistencia al aire y capturar la máxima potencia hasta llevarla a la posición bandera, es decir en forma paralela a la dirección de viento. La falla F6 se refiere al mecanismo de Yaw que controla el movimiento de la góndola del AG.

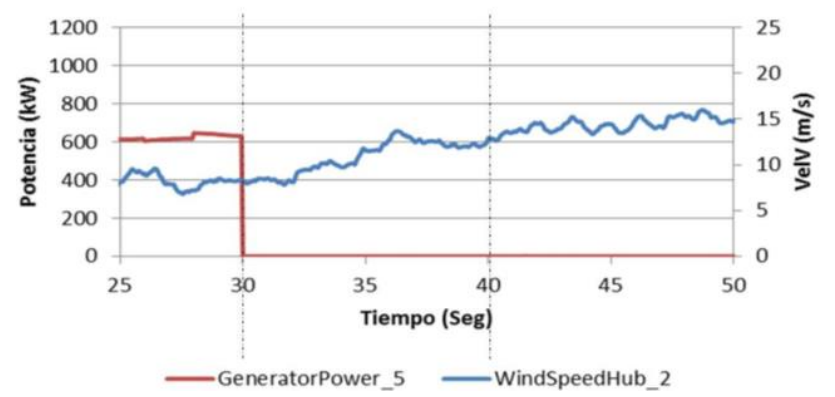

Figura 2 Simulación de la falla de un corto circuito Fuente: Elaboración Propia

En la Figura 2 se muestran dos variables del aerogenerador, en color rojo la generación de potencia y en color azul la velocidad de viento. Se puede apreciar la falla del aerogenerador ocasionada por un corto circuito, cuando la generación se pone en cero aún con viento favorable.

\section{Experimentos y resultados}

Para clasificar de manera correcta la falla raíz, se establece un procedimiento de reconocimiento de patrones, tal y como se muestra en la Figura 3 , que consiste en los siguientes 6 pasos:

i) Patrón de falla: Describe el conjunto de variables con comportamiento anormal que se produce a partir de una falla del AG. El patrón de falla se obtiene del sistema DxAG tal como se describe en la sección 2.

ii) Formación de objetos: Describe la extracción de características partiendo de un patrón de comportamiento de falla.

iii) Reducción de dimensionalidad: Es el proceso para obtener el mejor subconjunto de variables en un conjunto de datos mediante una búsqueda automática.

iv) Modelo de clasificación: Es el algoritmo aplicado al conjunto de patrones de fallas definidos para la clasificación de la falla raíz.

v) Evaluación del modelo: Es el conjunto de procesos para verificar que los modelos de clasificación se estén desempeñando de manera correcta.

vi) Clasificación de falla raíz: Establece la precisión y exactitud del modelo para clasificar futuras fallas.

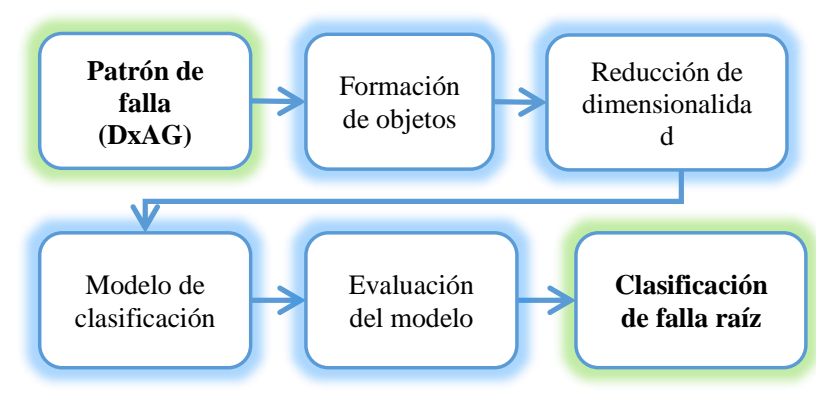

Figura 3 Procedimiento de reconocimiento de patrones

\section{Formación de objetos}

Partiendo de un patrón de falla (ver Figura 4) generado por el sistema DxAG, se continúa con el proceso de generar características presentes en los datos adquiridos de los patrones de cada falla, los cuales pueden ser pasados directamente a la etapa de clasificación de fallas. 


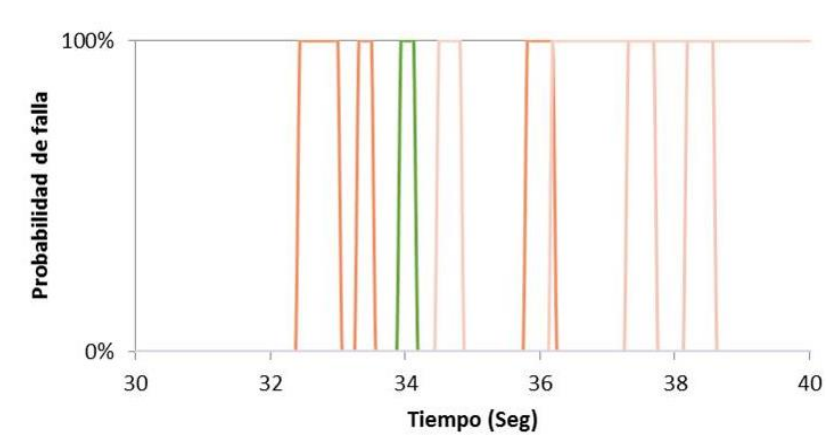

Figura 4 Patrón de la falla F1 Fuente: Elaboración Propia

El patrón de falla de la Figura 4, donde el eje de las " $x$ " representa el tiempo en segundos del conjunto de variables y el eje de las " $y$ " el porcentaje de probabilidad de falla, esto se toma como referencia, como una fotografía de la falla donde se involucra un conjunto variables en comportamiento anormal, tal como se describe en secciones anteriores. Tomando en cuenta que el objetivo del reconocimiento de patrones es asignar un patrón a la clase a la que pertenece, es necesario extraer características que permitan representarse y describirse como un vector de características cuantitativas globales. En la Figura 5 se describe una matriz de $m$ registros por $n$ variables involucrados en una falla a la cual se procede a generar un vector de $m \times n$ características cuantitativas donde $\mathrm{X}$ es el patrón y x son las características descriptivas.

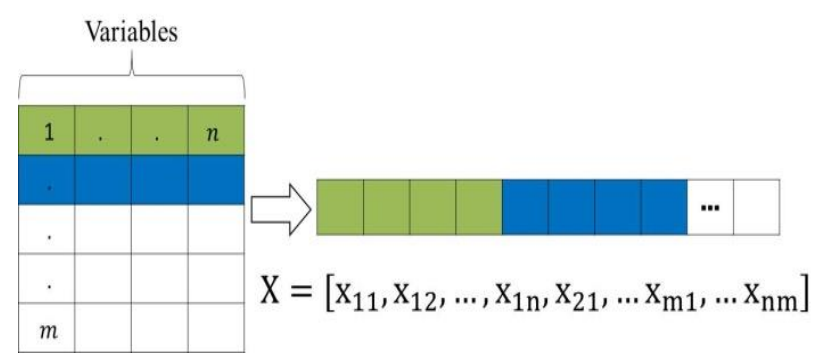

Figura 5 Vector de características cuantitativas Fuente: Elaboración Propia

La extracción de características, partiendo del patrón de falla, tiene como objetivo obtener un único un patrón de comportamiento de la falla a clasificar que contenga la mayor información representativa de ésta.

\section{Reducción de dimensionalidad}

La selección de características o variables importantes, es un proceso donde se busca obtener el mejor subconjunto de características dentro de un conjunto de datos mediante una búsqueda automática.
Por lo tanto, es necesario extraer aquellas variables que sean útiles para distinguir los patrones de cada falla. Además de que contribuye a mejorar la precisión del modelo de clasificación, ayuda a disminuir el tiempo de entrenamiento y colabora a obtener menos datos redundantes.

La selección de características mediante el algoritmo de aprendizaje "Random Forest" (RF) se implementa mediante algoritmos que tienen sus propios métodos de selección de características incorporados, por lo tanto, cuando se entrena un árbol, es posible calcular cuánto disminuye la entropía de cada variable; cuanto mayor es la disminución de la entropía, más significativa es la variable. Hacer una selección de variables mediante las características importantes derivadas de árboles es una forma muy sencilla, rápida $\mathrm{y}$ generalmente, precisa.

Para reducir la dimensionalidad de los datos, se creó un modelo de selección de características importantes basado en el algoritmo RF, posterior a ello, mediante una visualización de los datos (ver Figura 6). Se seleccionaron todas aquellas variables con puntaje mayor a cero logrando una reducción del $37 \%$ sobre el total de las variables seleccionadas.

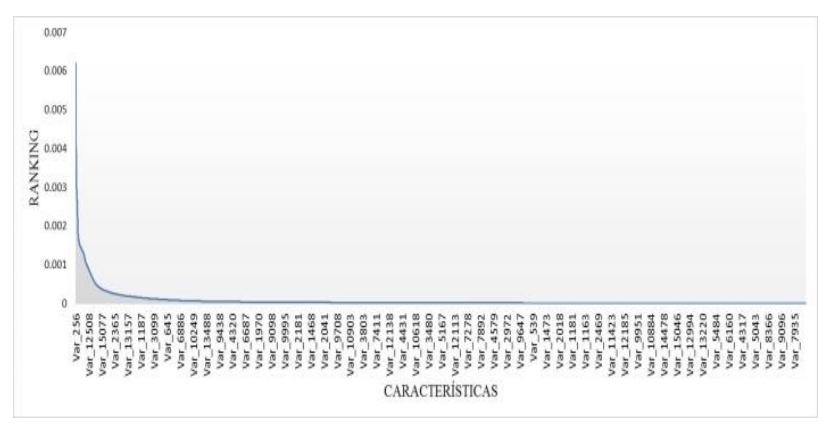

Figura 6 Características importantes. En el eje de las "x" se describen las variables a evaluar y en el eje de las " $y$ " se describe la importancia de la variable

Fuente: Elaboración Propia

\section{Modelo de clasificación}

Dentro de los algoritmos supervisados del aprendizaje automático se encuentran los de clasificación, que tiene como objetivo el poder saber a qué clase pertenece una observación dada. El algoritmo de RF es uno de los más populares y estudiados en cuanto a clasificación. En general, brindan un buen desempeño gracias al ensamble de muchos árboles de decisión. 
El algoritmo consiste en un gran número de árboles de decisión aleatorios, cada árbol de decisión en el bosque aleatorio genera una predicción de clase y la clase con la mayor cantidad de votos se convierte en la predicción del modelo (vea Figura 7). En general, mientras más árboles existan en el bosque, más robusto podría ser el algoritmo.

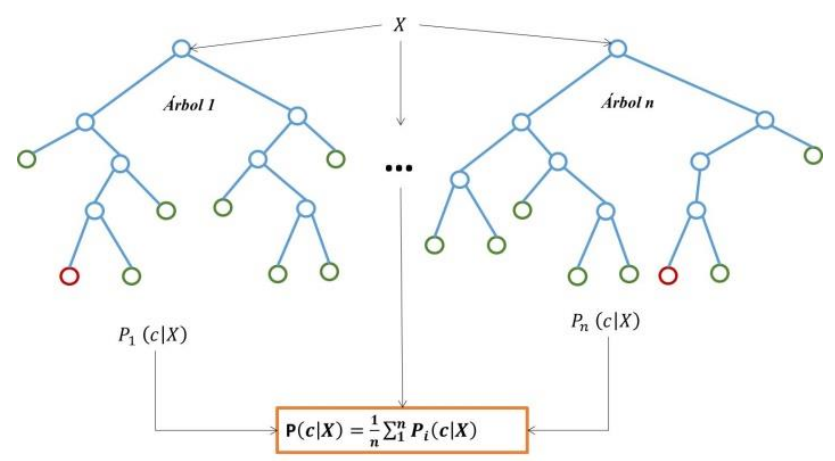

Figura 7 Algoritmo de clasificación: "Random Forest" Fuente: Elaboración Propia

La Figura 7 muestra una explicación general del funcionamiento del algoritmo como clasificador con un número $n$ de árboles de decisión, donde $\mathrm{X}$ son las características seleccionadas y c es la predicción de la clase.

La capacidad de clasificar con una buena precisión las observaciones dadas, es de vital importancia para diversas aplicaciones en el sector energético, en particular para clasificar fallas tipo raíz de los AGs.

\section{Evaluación del modelo}

Para determinar si un modelo de Aprendizaje Automático realizará un buen trabajo de clasificación con futuras fallas, es importante evaluarlo. Dado que las futuras fallas tienen valores desconocidos a los previamente entrenados, debe evaluarse el modelo mediante distintas métricas. Por ejemplo, precisión, exactitud y recuperamiento (recall) (Hossin \& Sulaiman, 2015) con respecto a los datos de fallas conocidas. Esto con el objetivo de tener una medida del desempeño de nuestro modelo para clasificar futuras fallas.

Para poder evaluar un modelo correctamente, se debe tener una muestra de datos que sea etiquetada como datos de entrenamiento para el modelo de clasificación y otra muestra de datos para probar el modelo distinto a los datos etiquetados como entrenamiento.
Las siguientes métricas se usaron para evaluar y validar los modelos de clasificación.

Exactitud (acc): Mide la bondad de un modelo de clasificación como la proporción de los resultados verdaderos al total de casos.

$\mathrm{acc}=\frac{\mathrm{tp}+\mathrm{tn}}{\mathrm{tp}+\mathrm{tn}+\mathrm{fp}+\mathrm{fn}}$

Precisión (p): Es la proporción de resultados verdaderos sobre todos los resultados positivos.

$\mathrm{p}=\frac{\mathrm{tp}}{\mathrm{tp}+\mathrm{fp}}$

Recall (r): Es la fracción de todos los resultados correctos devueltos por el modelo.

$r=\frac{t p}{t p+f n}$

La validación cruzada: Se utilizó esta técnica para evaluar el rendimiento del modelo de clasificación mediante un procedimiento básico de 4 pasos:

i) Se dividió el conjunto de datos en 2 partes etiquetando el primero como datos de entrenamiento y el segundo como prueba.

ii) Se entrenó el modelo con el primer conjunto de datos

iii) Se evaluó el modelo con el conjunto de pruebas

iv) Para evitar sesgos en la prueba el modelo se entrenó y evaluó durante 10 veces, repitiendo los pasos 1-3.

Matriz de confusión: Describe el rendimiento del modelo de clasificación RF dado un conjunto de datos de prueba para los cuales se conocen los valores reales (ver Figura 8).

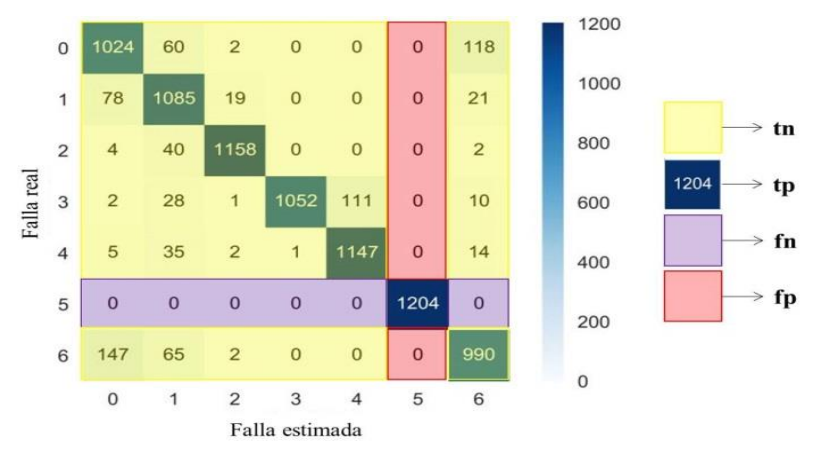

Figura 8 Matriz de confusión

Fuente: Elaboración Propia 
La Figura 8 se describe el rendimiento de clasificación de múltiples fallas, en el eje de las " $x$ " se representa las fallas estimadas por el algoritmo y en el eje de las "y" representa las fallas reales que se sometieron a evaluación.

Dónde:

- $\quad$ tp (verdaderos positivos): Es el número de casos que clasificó correctamente la clase positiva.

- fn (falsos negativos): Es el número de casos que clasificó incorrectamente la clase negativa.

- $\quad$ fp (Falsos positivos): Es el número de casos que clasificó incorrectamente la clase positiva.

- $\quad$ tn (verdaderos negativos): Es el número de casos que clasificó correctamente la clase negativa.

En la Tabla 2 se describe el resultado de la evaluación del algoritmo RF para clasificar múltiples fallas, donde se destaca el porcentaje de precisión y recall de cada una de las fallas evaluadas.

\begin{tabular}{|l|r|r|}
\hline \multicolumn{3}{|c|}{ Reporte de la clasificación } \\
\hline \multicolumn{1}{|c|}{ Clase } & Precisión (\%) & \multicolumn{1}{c|}{ Recall (\%) } \\
\hline Falla0 & 82 & 85 \\
\hline Falla1 & 83 & 91 \\
\hline Falla2 & 98 & 97 \\
\hline Falla3 & 99 & 87 \\
\hline Falla4 & 91 & 96 \\
\hline Falla5 & 99 & 100 \\
\hline Falla6 & 86 & 83 \\
\hline
\end{tabular}

Tabla 2 Tabla de resultados

Fuente: Elaboración Propia

\section{Clasificación de la falla raíz}

Con base en los resultados expuestos se demuestra que el algoritmo "Random Forest" presenta un buen rendimiento para clasificar distintas fallas simuladas en la MEM, con el $91.14 \%$ de exactitud y un $91 \%$ de precisión del modelo generado. Se comprueba que tendrá un buen rendimiento con las nuevas fallas que no hayan sido usadas para el entrenamiento del modelo de clasificación de fallas múltiples.En la Figura 9 se describe en términos de porcentaje el resultado de la clasificación de cada falla raíz evaluada. Por ejemplo, para F1 (falla real) el modelo clasifica la falla como F1 (falla estimada) con el $90.2 \%$, con el $6.5 \%$ lo clasifica como F0, con el $1.6 \%$ lo clasifica como F2 y con el $1.7 \%$ lo clasifica como F6.
La variación de porcentaje con el cual se clasifica las fallas se debe en gran parte al comportamiento de operación similar que existe entre ellas. En estos experimentos podemos observar que la F5 fue clasificada con el $100 \%$ debido a que es una falla que no presenta un comportamiento de operación similar con el resto de las fallas por tratarse de un corto circuito en el generador.

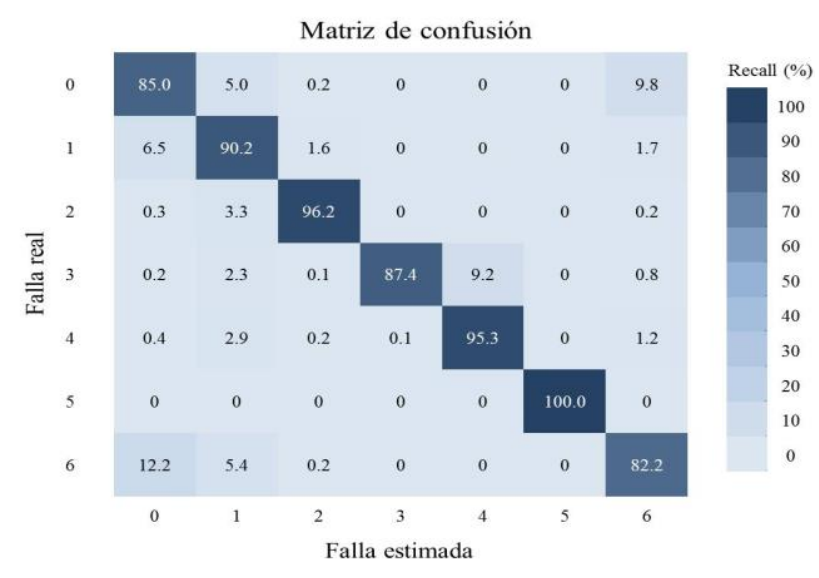

Figura 9 Clasificación de la falla raíz

Fuente: Elaboración Propia

\section{Agradecimiento}

Este proyecto fue realizado con el apoyo del CEMIE-Eólico del Fondo CONACYT-SENERSustentabilidad y del proyecto 14629 del INEEL. Agradecimiento especial al Ing. Fortino Mejía Neri del INEEL, por su apoyo con el programa de simulación Focus6.

\section{Conclusiones}

Se presenta en este artículo el desarrollo de un sistema de diagnóstico de aerogeneradores utilizando técnicas de IA. El diagnóstico consiste en dos etapas. Primero, se utiliza el DxAG, un sistema diseñado por este grupo de trabajo que utiliza modelos probabilistas de comportamiento para generar patrones de comportamiento anormal. Segundo, el módulo presentado en este artículo que toma los patrones generados e identifica la falla raíz que causó el mal comportamiento del AG. Esta identificación de falla se realiza utilizando técnicas de aprendizaje automático. Para entrenar y probar el sistema, se corrieron experimentos en el simulador de la Máquina Eólica Mexicana diseñada en el INEEL. Los resultados muestran que el algoritmo "Random Forest" mantiene una precisión y exactitud muy aceptables para esta aplicación. Como trabajo futuro se tiene las pruebas en línea en algún $\mathrm{AG}$ en un parque eólico.

GARCÍA, Uriel A., IBARGÜENGOYTIA, Pablo H., DÍAZ GONZÁLEZ, Lorena y HERMOSILLO-VALADEZ, Jorge. Técnicas de aprendizaje automático en el diagnóstico de aerogeneradores. Revista de Energías Renovables. 2019 


\section{Referencias}

WMC. (26 de 01 de 2010). Recuperado el 01 de 03 de 2018, de WMC: https://wmc.eu/focus6.php

AMDEE. (20 de Septiembre de 2017). Recuperado el 30 de Mayo de 2019, de La Asociación Mexicana de Energía Eólica A.C "AMDEE": http://www.amdee.org

Arteaga Celedonio, A. (2019). Estrategias de eficiencia energética en el diseño de un centro empresarial en Pacasmayo (Tesis parcial).

Christopher M., B. (2006). Pattern Recognition and Machine Learning. New York: Springer.

García Márquez, F., MarkTobias, A., Pinar Pérez, J., \& Papaelias, M. (2012). Condition monitoring of wind turbines: Techniques and methods. ELSEVIER, 169-178.

García, U. A., Ibargüengoytia, P. H., Reyes, A., $\&$ Borunda, M. (18 de 05 de 2016). Modelo de Comportamiento de una turbine eólica. Congreso Mexicano de Inteligencia Artificial, págs. 119-129.

González González, G. L. (2019). Pronóstico de generación eléctrica de corto plazo en parques eólicos utilizando técnicas de predicción de clima de microescala.

Gutiérrez, J., Flórez, L., \& Serrano, D. (2017). Convertidor dc-ac de alta eficiencia basado en el modulador EG8010 para implementar en sistemas energéticos alternativos. Revista Publicaciones e Investigació, 141-14.

Hossin, H., \& Sulaiman, M. (Marzo de 2015). A review on evaluation metrics for data classification evaluations. International Journal of Data Mining \& Knowledge Management Process (IJDKP).

Ian H., W., \& Eibe, F. (2005). Data Mining: Practical Machine Learning Tools and Techniques. USA: Morgan Kaufmann.

Ibargüengoytia, P., García, U., Reyes, A., \& Borunda, M. (2016). Anomalies Detection in the Behavior of Processes Using the Sensor Validation Theory. IBERAMIA 2016 (págs. 1424). San Jose, Costa Rica: Springer.
Ibargüengoytia, P., Vadera, S., \& Sucar, L. (2006). A Probabilistic Model for Information and Sensor Validation. The Computer Journal, 113-126.

Lopez, E., Garcia, U., Ibargüengoytia, P., \& Serrano, L. (2016). Evaluation of Artificial Intelligence Techniques for Vibration Analysis of Turbogenerators. Congreso Internacional en Innovación y Desarrollo Tecnológico, CIINDET 2016. Cuernavaca, Mor.: IEEE.

Meik, S., Ferreira Santos, i., \& Achiche, S. (2013). Wind turbine condition monitoring based on SCADA data using normal behavior models. Part 1: System description. ELSEVIER, 259-270.

Navarro, V., \& José Raúl. (2019). Estudio técnico y económico, de la utilización de energía eólica y solar, para generar energía eléctrica en el caserío La Lima distrito Santo Tomas Cutervo-Cajamarca.

Pearl, J. (1988). Probabilistic Reasoning in Intelligent Systems: networks of plausible inferenc. Palo Alto, Calif: Morgan Kaufmann.

Peter, F. (2012). MACHINE LEARNING: The Art and Science of Algorithms that Make Sense of Data. New York: CAMBRIDGE.

S. K., A., K. G., O., F. V., J., \& F., J. (1989). Hugin - a shell for building Bayesian belief universes for expert systems. In Proceedings of the Eleventh International Joint Conference on Artificial Intelligence, 1080-1085.

Sepúlveda, C., Javier, M., Urrutia, P., \& paz, J. (2019). Prefactibilidad de instalación de iluminaria en parque quebrada verde, mediante uso de paneles fotovoltaicos y uso de aerogeneradores.

Stuart, R., \& Peter, N. (2010). Artificial Intelligence. New Jersey: PEARSON.

Tom M., M. (1997). Machine Learning. Ithaca, NY: McGraw-Hill.

Zhou, A., Yu, D., \& Zhang, W. (2015). A research on intelligent fault diagnosis of wind turbines based on ontology and FMECA. ELSEVIER, 115-125. 